\title{
Coded Ultrasound for Blood Flow Estimation Using Subband Processing
}

\author{
Gran, Fredrik; Udesen, Jesper; Nielsen, Michael Bachamnn; Jensen, Jørgen Arendt
}

Published in:

IEEE Transactions on Ultrasonics Ferroelectrics and Frequency Control

Link to article, DOI:

10.1109/TUFFC.920

Publication date:

2008

Document Version

Publisher's PDF, also known as Version of record

Link back to DTU Orbit

Citation (APA):

Gran, F., Udesen, J., Nielsen, M. B., \& Jensen, J. A. (2008). Coded Ultrasound for Blood Flow Estimation Using Subband Processing. IEEE Transactions on Ultrasonics Ferroelectrics and Frequency Control, 55(10), 22112220. https://doi.org/10.1109/TUFFC.920

\section{General rights}

Copyright and moral rights for the publications made accessible in the public portal are retained by the authors and/or other copyright owners and it is a condition of accessing publications that users recognise and abide by the legal requirements associated with these rights.

- Users may download and print one copy of any publication from the public portal for the purpose of private study or research.

- You may not further distribute the material or use it for any profit-making activity or commercial gain

- You may freely distribute the URL identifying the publication in the public portal 


\title{
Coded Ultrasound for Blood Flow Estimation Using Subband Processing
}

\author{
Fredrik Gran, Jesper Udesen, Michael Bachmann Nielsen, \\ and Jørgen Arendt Jensen, Senior Member, IEEE
}

\begin{abstract}
This paper investigates the use of coded excitation for blood flow estimation in medical ultrasound. Traditional autocorrelation estimators use narrow-band excitation signals to provide sufficient signal-to-noise-ratio (SNR) and velocity estimation performance. In this paper, broadband coded signals are used to increase SNR, followed by subband processing. The received broadband signal is filtered using a set of narrow-band filters. Estimating the velocity in each of the bands and averaging the results yields better performance compared with what would be possible when transmitting a narrow-band pulse directly. Also, the spatial resolution of the narrow-band pulse would be too poor for brightness-mode (Bmode) imaging, and additional transmissions would be required to update the B-mode image.

For the described approach in the paper, there is no need for additional transmissions, because the excitation signal is broadband and has good spatial resolution after pulse compression. This means that time can be saved by using the same data for B-mode imaging and blood flow estimation. Two different coding schemes are used in this paper, Barker codes and Golay codes. The performance of the codes for velocity estimation is compared with a conventional approach transmitting a narrow-band pulse. The study was carried out using an experimental ultrasound scanner and a commercial linear array $7 \mathrm{MHz}$ transducer. A circulating flow rig was scanned with a beam-to-flow angle of $60^{\circ}$. The flow in the rig was laminar and had a parabolic flow-profile with a peak velocity of $0.09 \mathrm{~m} / \mathrm{s}$. The mean relative standard deviation of the velocity estimate using the reference method with an 8-cycle excitation pulse at $7 \mathrm{MHz}$ was $0.544 \%$ compared with the peak velocity in the rig. Two Barker codes were tested with a length of 5 and 13 bits, respectively. The corresponding mean relative standard deviations were $0.367 \%$ and $0.310 \%$, respectively. For the Golay coded experiment, two 8-bit codes were used, and the mean relative standard deviation was $0.335 \%$.
\end{abstract}

\section{INTRODUCTION}

$\mathrm{I}_{\mathrm{r}}^{\mathrm{\Upsilon}}$ N conventional Doppler ultrasound, several narrowband pulses are transmitted in the direction in which the blood velocity is to be estimated. Data lines are thereafter beamformed, and the scanned object is imaged. To determine the velocity at a specific depth in the image, the beamformed data are sampled at this depth over the acquired data lines, creating a slow-time signal. The blood

Manuscript received January 6, 2008; accepted May 7, 2008. This work was supported by the Danish research council for Technology and Production, grant: 274-05-0327 and by B-K Medical ApS, Denmark.

F. Gran, J. Udesen, and J. A. Jensen are from the Center for Fast Ultrasound Imaging, Ørsted•DTU, Building 348, Technical University of Denmark, Lyngby, Denmark (e-mail: fg@oersted.dtu.dk).

M. B. Nielsen is from the Section of Ultrasound, Department of Radiology, University Hospital of Copenhagen, Copenhagen, Denmark.

Digital Object Identifier 10.1109/TUFFC.920 velocity is typically estimated using an autocorrelation estimator [1].

The advantage of using narrow-band pulses in transmit is 2-fold:

- A longer sinusoidal excitation waveform (narrow band) has more energy than a shorter signal and will provide a better signal-to-noise-ratio (SNR).

- The variance of the autocorrelation estimate decreases with increasing pulse duration [2].

However, longer pulse duration results in poor axial resolution, and the generated data lines cannot be used for B-mode imaging. Therefore, a separate B-mode sequence has to be transmitted with a shorter (broadband) excitation that decreases the total frame rate.

In [3] it was suggested that a broadband signal be used as excitation. The received signal is separated into several frequency bands with the same bandwidth as that of a conventional Doppler pulse. Thereby, several flow estimates can be estimated for the same time period as a conventional system would require for estimating only one line. The estimates can thereafter be averaged to yield a lower variance on the final velocity estimate.

This was further investigated in [4] where it was suggested that a nonlinear frequency-modulated excitation signal and subsequent subband processing be used. If a short sinusoidal excitation is used, the SNR would be insufficient. Therefore, it was suggested that a nonlinear frequency modulated (NLFM) signal [5]-[8] be used to excite the transducer. However, to obtain a sufficient sidelobe level of the NLFM, long sequences (10 to $20 \mu \mathrm{s}$ ) have to be used. Therefore, the transmitted energy will be increased drastically compared with a conventional Doppler pulse under the same excitation voltage. In combination with a focused transmission and a high-pulse repetition frequency $f_{\text {prf }}$, the generated intensities in tissue will violate the regulations set by the Food and Drug Administration (FDA) [9].

This paper investigates the possibility of using other code sequences to increase SNR as compared with a short broadband pulse without exceeding the intensity generated by a long narrowband Doppler pulse (4 to 10 cycles). Two different binary coding schemes are investigated and compared with conventional Doppler:

- Barker codes

- Complementary (Golay) codes 
The Barker sequences [10] are biphase sequences with autocorrelation functions satisfying

$$
\begin{gathered}
r_{c}(0)=1 \\
r_{c}(m) \leq \frac{1}{N}, \quad m \neq 0 .
\end{gathered}
$$

Here

$$
r_{c}(m)=\frac{1}{N} \sum_{n=0}^{N-1} c(n) c(n+m),
$$

is the autocorrelation function of the $N$-bit code sequence $c(n)$. Matched filtering seems to be an obvious solution for pulse compression. Unfortunately, the longest known Barker code is 13 bit, restricting the match-filtered Barker codes to have a range sidelobe level above $-22.3 \mathrm{~dB}$. This is not sufficient for ultrasound imaging where the dynamic range is between 40 to $100 \mathrm{~dB}$. In this paper, the decoding filters are designed as finite impulse response (FIR) filters using a fast Fourier transform (FFT) [11], [12] based approach. This makes it possible to lower the sidelobe level to below $-80 \mathrm{~dB}$ below the mainlobe of the compressed code.

Golay sequences have been suggested for use in ultrasound imaging [13]. These sequences satisfy the so-called complementary condition. A set of sequences is said to be complementary when the sum of the autocorrelation functions of the sequences is a perfect digital delta function

$$
\frac{1}{N} \sum_{n=0}^{N-1}\left\{c_{1}(n) c_{1}(n+m)+c_{2}(n) c_{2}(n+m)\right\}=2 \delta(m)
$$

where $c_{1}(n)$ is the first sequence and $c_{2}(n)$ is the second sequence. Pulse compression is achieved by transmitting $c_{1}(n)$ in the first transmission and $c_{2}(n)$ in the second transmission and correlating the received signals with the corresponding codes and summing the result. For this approach to work without artifacts, the target has to be completely stationary between the 2 transmissions. If motion is present, the compressed output will be degraded, with loss in contrast as a result.

This paper investigates coded broadband ultrasound for blood velocity estimation. The velocity is estimated by dividing the received signal into different subbands and estimating the velocity in each of the bands. Thereby, the number of velocity estimates per time unit increases. The final velocity estimate is formed by averaging the velocity estimates in each of the bands yielding a better estimate. Both Barker and Golay codes are investigated.

The paper is structured as follows: In Section II, the designs of the Barker and Golay codes are discussed, and decoding is reviewed. In Section III, the subband processing and velocity estimation is explained. In Section IV, performance is evaluated by measurements in a flow rig. The flow profiles, obtained with the coded methods, are compared with those of a conventional system with a narrow-band excitation, and the variance of the estimates is assessed. In Section VI, the results are analyzed and discussed.

\section{Code And Waveform Design}

This section describes the 2 coding schemes used in the paper.

\section{A. Barker Codes}

An $N$-bit Barker code is denoted

$$
\mathbf{c}=\left(\begin{array}{llll}
c(0) & c(1) & \cdots & c(N-1)
\end{array}\right)
$$

In this paper a 5-bit and a 13-bit code are used

$$
\begin{gathered}
\mathbf{c}_{5}=\left(\begin{array}{lllllllllll}
1 & 1 & 1 & -1 & 1
\end{array}\right) \\
\mathbf{c}_{13}=\left(\begin{array}{lllllllllllll}
1 & 1 & 1 & 1 & 1 & -1 & -1 & 1 & 1 & -1 & 1 & -1 & 1
\end{array}\right) .
\end{gathered}
$$

To obtain a waveform which can easily be transmitted by the transducer, the bit-rate of the code is first decreased, and the resulting code is thereafter convolved with a chipwaveform $p(n)$

$$
s(n)=\sum_{l=0}^{N-1} c(l) p\left(n-l \cdot T f_{s}\right),
$$

where $T$ is the time duration between bits, $f_{s}$ is the sampling frequency of the ultrasound system, and $p(n)$ is a chip waveform.

Ideally, the code sequence $c(n)$ has amplification in all frequencies. By reducing the bit rate of the code, the spectrum of the original code will be confined to frequencies in the range $[-1 /(2 T), 1 /(2 T)]$. However, due to aliasing, the spectrum will be replicated at intervals of $p(1 / T) \mathrm{Hz}$, where $p$ is an integer. So naturally, the code sequence with reduced bit rate will also have amplification in all frequencies.

Therefore, applying the chip, not only increases the total transmitted energy, the total bandwidth of the waveform is also reduced. By choosing the chip carefully, the final waveform $s(n)$ can be designed so that it has most of the energy confined in the transducer passband. This may avoid unnecessary heating of the transducer.

Typically, matched filtering is used for compressing the code at the receiver, however, the longest known Barker code is 13 bit, restricting the match-filtered Barker codes to have a range sidelobe level above $-22.3 \mathrm{~dB}$. This is not sufficient for ultrasound imaging, and in this paper, sufficient range lobe level is achieved by designing FIR filters approximating the inverse filter of the code sequence. The 
first step toward designing a FIR decoding filter is to calculate the Fourier transform of the code,

$$
C(f)=\sum_{n=0}^{N-1} c(n) e^{-j 2 \pi\left(f / f_{s}\right) n}, \quad-\frac{f_{s}}{2} \leq f \leq \frac{f_{s}}{2} .
$$

The objective will be to derive an $L$ tap FIR filter whose spectrum is the inverse of that in (9). The desired spectrum is, therefore,

$$
D(f)=\frac{1}{C(f) e^{j \pi\left(f / f_{s}\right) N}} \cdot e^{-j \pi\left(f / f_{s}\right) L}, \quad-\frac{f_{s}}{2} \leq f \leq \frac{f_{s}}{2} .
$$

The factor $e^{j \pi\left(f / f_{s}\right) N}$ in the denominator in (10) is applied to remove the linear phase resulting from the time delay of $N / 2$ in (5). Correspondingly, $e^{-j \pi(f / f s) L}$ is applied to generate a phase on the filter that corresponds to a delay of $L / 2$ samples. The inversion of (9) is allowed, because the spectrum of the Barker code contains no zeros. The spectrum is evaluated for $M$ frequency points in the range $\left[-f_{s} / 2, f_{s} / 2\right]$ to form the vector

$$
\mathbf{D}=\left(\begin{array}{llll}
D\left(f_{0}\right) & D\left(f_{1}\right) & \cdots & D\left(f_{M-1}\right)
\end{array}\right)^{T},
$$

where $(\cdot)^{T}$ is the transpose of $(\cdot)$.

Barker codes with duration over 2 bits all share the property that they have spectral support in the entire range (no frequencies have zero amplification) [10]. However, there is a variation in the amplitude of the spectrum so that the spectrum will not have a flat response. When the decoding filter is applied, it is desirable that the filter equalizes the ripples in the spectrum of the code. Using an ideal filter would result in a completely flat spectrum after decoding, effectively compressing the code. With the properties of the Barker codes in mind, inverse filtering [14] in the frequency domain would be an obvious solution. However, to make the decoding more tractable from a computational perspective, an $L$ tap FIR pseudo-inverse filter is designed.

The $L$ tap FIR filter, which minimizes the error between the calculated spectrum and the desired spectrum in the least squares sense, is given by the following minimization problem [7]

$$
\hat{\mathbf{h}}=\arg \min _{\mathbf{h}}\|\mathcal{W} \mathbf{h}-\mathbf{D}\|^{2}
$$

where

$$
\mathbf{h}=\left(\begin{array}{llll}
h(0) & h(1) & \cdots & h(L-1)
\end{array}\right)
$$

and $\hat{\mathbf{h}}$ is defined correspondingly. The matrix $\mathcal{W}$ is the $M$ $\times L$ Fourier matrix with elements defined as

$$
\mathcal{W}_{m l}=e^{-j 2 \pi(l m / M)}, \quad \begin{aligned}
l & =0, \ldots, L-1 \\
m & =0, \ldots, M-1
\end{aligned} .
$$

The solution to (12) is given by [15]

$$
\hat{\mathbf{h}}=\left(\mathcal{W}^{H} \mathcal{W}\right)^{-1} \mathcal{W}^{H} \mathbf{D}
$$

The operator $(\cdot)^{H}$ is the complex conjugate transpose of $(\cdot)$.

The unique solution to (15) exists if $\mathcal{W}$ has full column rank. The columns of a Fourier matrix will be orthogonal as long as $M \geq L$. Therefore, as long as there are more frequency points in the desired spectrum than samples in the FIR filter, the optimization problem will have a unique solution. If the orthogonality condition is satisfied,

$$
\mathcal{W}^{H} \mathcal{W}=M \mathbf{I}
$$

where $\mathbf{I}$ is the identity matrix. Therefore, the solution in (15) reduces to

$$
\hat{\mathbf{h}}=\frac{1}{M} \mathcal{W}^{H} \mathbf{D} .
$$

If one of the samples in $\hat{\mathbf{h}}$ is analyzed

$$
\hat{h}(l)=\frac{1}{M} \sum_{m=0}^{M-1} D\left(f_{m}\right) e^{j 2 \pi(l m / M)}, \quad l=0, \ldots, L-1,
$$

This means that the optimal FIR filter in the least squares sense is the inverse discrete Fourier transform of the sampled desired frequency spectrum of the filter. In terms of implementation, this can be interpreted as taking the $L$ first samples of the inverse FFT of the sampled desired spectrum. This has previously been suggested in [16] and [17].

Because the code consists both of the code and the chip, the filter also has to undergo the same transformation as the code that is decreasing the bit rate to fit the chip waveform. By doing this, the effective sampling frequency will be the same for the decreased bit-rate filter and the decreased bit-rate code: $1 / T$.

Therefore, the final decoding filter is the convolution of the time-reversed chip-waveform and decreased bit-rate version of $h(n)$

$$
g(n)=\sum_{l=0}^{L-1} h(l) p\left(-n+l \cdot T f_{s}\right)
$$

Note that it is not necessary to apply the time reversed chip to achieve pulse compression. The time-reversed chip merely serves as a matched filter for the chip in the transmitted waveform and effectively acts as a bandpass filter.

Two examples are shown in Fig. 1 and 2 where a 5-bit and a 13-bit Barker code are used with a chip waveform consisting of a single cycle sinusoid at $7 \mathrm{MHz}$. The bottom plots illustrates the log-compressed envelope of the decoded output when using (19) for decoding filters with 


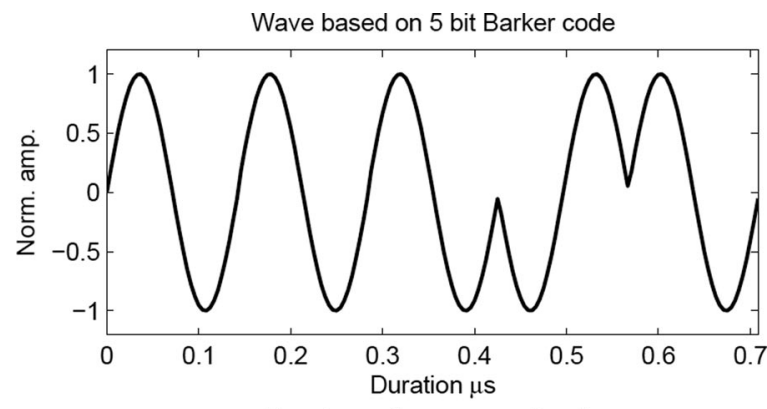

Envelope of compressed code

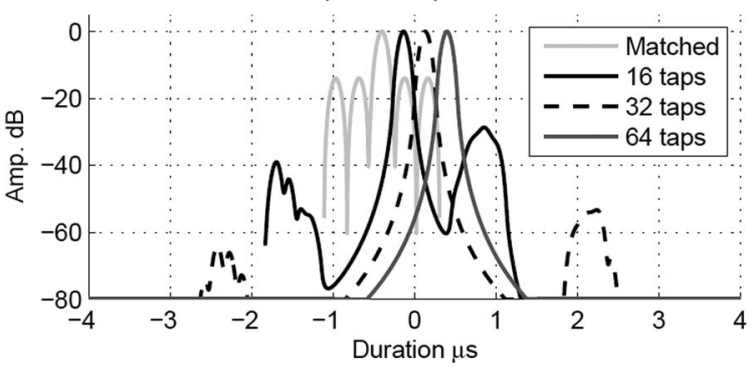

Fig. 1. The top plot displays the 5-bit Barker code convolved with the chip waveform $p(n)$. The bottom plot shows the envelope of the code after compression with different filter lengths. The responses are shifted in time to facilitate visualization. The matched filtered response is also given to serve as a reference.

lengths $L=16,32$, and 64 , respectively. Note that the responses are shifted in time to facilitate visualization.

\section{B. Golay Codes}

The investigation also included use of complementary sequences. Two complementary 8-bit codes used in this work are given by

$$
\begin{gathered}
\mathbf{c}_{1}=\left(\begin{array}{llllllll}
1 & 1 & 1 & -1 & 1 & 1 & -1 & 1
\end{array}\right) \\
\mathbf{c}_{2}=\left(\begin{array}{llllllll}
1 & 1 & 1 & -1 & -1 & -1 & 1 & -1
\end{array}\right) .
\end{gathered}
$$

These codes were generated using the Hadamard matrix as basis and by extending the code length as outlined by Chiao and Thomas [18]. The complementary property ensures that the sum of the autocorrelation functions of the 2 codes completely vanish for all lags except for lag zero. This means that for every data line, 2 transmissions have to be carried out before pulse compression can be achieved. This decreases the frame rate by a factor of 2 . However, the loss in frame rate can be recovered by orthogonal complementary sequences [18], and furthermore, under the assumption of stationarity, perfect compression is obtained. If this assumption is violated, the compression will be degraded, resulting in poor contrast. This is investigated briefly in Section II-B-1.

The transmitted waveforms were, as for the Barker code, a convolution of a chip waveform and the code sequences with decreased bit rate

$$
s_{i}(n)=\sum_{l=0}^{L-1} c_{i}(l) p\left(n-l \cdot T f_{s}\right), \quad i=1,2 .
$$
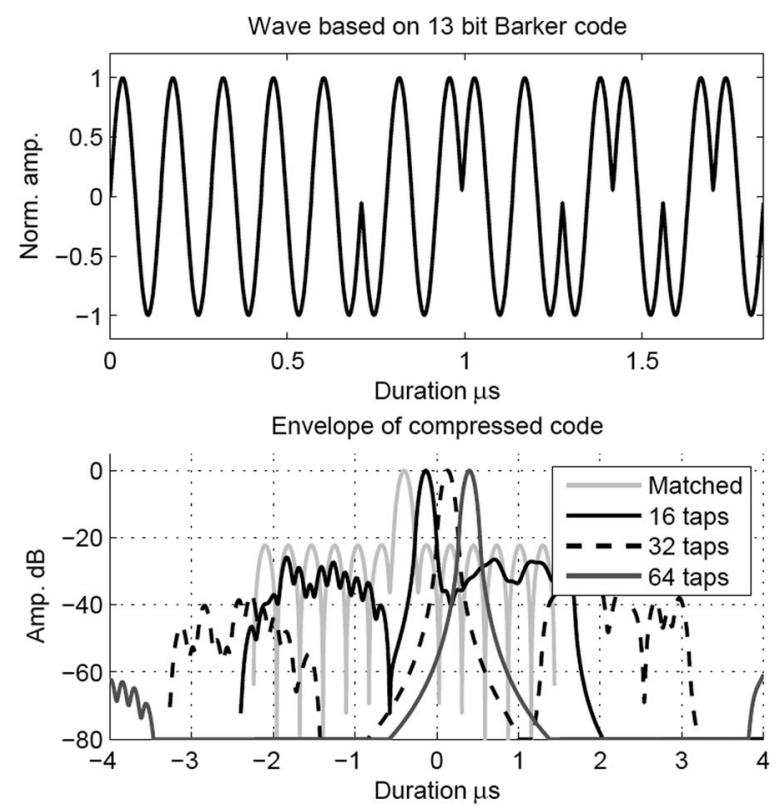

Fig. 2. The top plot displays the 13-bit Barker code convolved with the chip waveform $p(n)$. The bottom plot shows the envelope of the code after compression. The responses are shifted in time to facilitate visualization. The matched filtered response is also given to serve as a reference.

The 2 waveforms can be viewed in the top and the middle plots in Fig. 3, and the compressed result (under stationarity) is given as the bottom plot.

It is of interest to investigate how pulse compression of the Golay codes is degraded when the target is moving. This is of special importance when flow applications are considered, because blood can exhibit very rapid motion. When axial flow is examined, the sampled slow-time signal for a specific depth has a center frequency

$$
f_{D}=\frac{2 v_{z} f_{c}}{c},
$$

where $v_{z}$ is the axial velocity, $f_{c}$ is the center frequency of the emitted waveform, and $c$ is the speed of sound. Absolute velocities are of little interest here. The important thing to investigate is the velocity in comparison to the $f_{p r f}$. Normally, the pulse repetition frequency is adjusted so that $f_{D} \approx \pm f_{\mathrm{prf}} / 4$. The center frequency of the emitted waveforms was just as before $7 \mathrm{MHz}$, and the speed of sound was $1540 \mathrm{~m} / \mathrm{s}$. Four different velocities were investigated. The velocities were adjusted so that $f_{D}=$ $\left[0 f_{\text {prf }} / 10 f_{\text {prf }} / 4 f_{\text {prf }} / 2\right]$. The highest velocity is really extreme because this is the aliasing limit for the autocorrelation estimator, and this would always be avoided in a realistic implementation. The compressed code sequences can be viewed in Fig. 4, with the stationary result in the top and highest velocity in the bottom. The gray curve indicates the envelope of a match-filtered 8-cycle $7 \mathrm{MHz}$ pulse. It can be seen that the sidelobe level is drastically increased even at relatively small velocities $\left(f_{D}=f_{\mathrm{prf}} / 10\right)$, and at the highest velocity, no distinct peak can be distinguished. However, it should be noted that the axial re- 
Wave based on $c_{1}(n)$

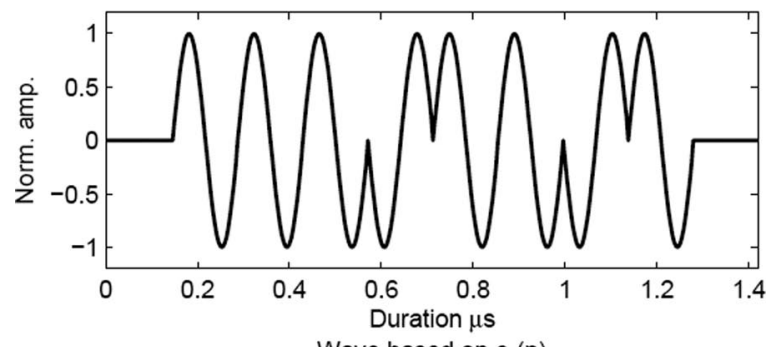

Wave based on $c_{2}(n)$

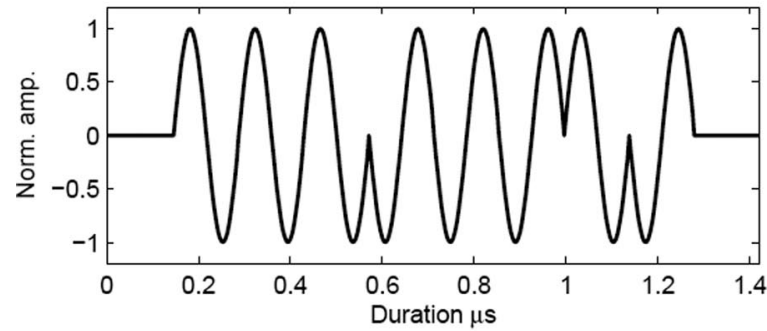

Envelope of compressed code

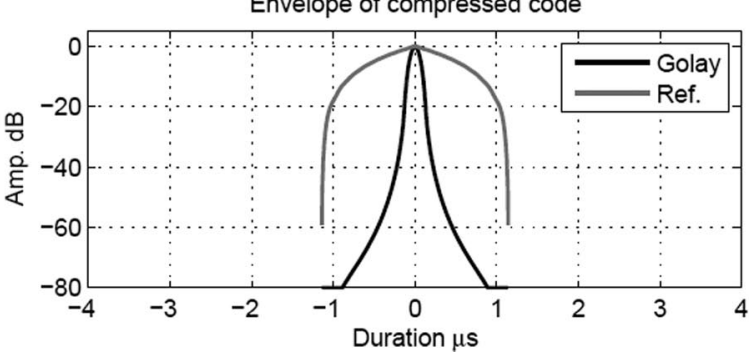

Fig. 3. The top and the middle plot represent the 2 complementary waveforms. The bottom plot is the envelope after pulse compression (under stationarity). Only the chip waveform remains. The gray curve indicates the envelope of the autocorrelation of the 8-cycle sinus used as a reference.

sponse of the Golay codes never exceeds that of the 8-cycle match-filtered response.

\section{Reference Method}

The 2 coded methods were compared with a conventional autocorrelation method. The excitation waveform was an 8-cycle sinusoidal signal at the center frequency of the transducer $(7 \mathrm{MHz})$. On reception of the scattered and reflected waves, the signals were matched-filtered with the time-reversed excitation signal to improve SNR and spectral resolution.

\section{Subband Processing and Velocity Estimation}

\section{A. Subband Processing}

Regardless of which coding scheme is used, a short broadband signal remains after pulse compression. To use the autocorrelation estimator, it is favorable to reduce the bandwidth of the measured signal. However, to exploit all the energy in the spectral support of the signal, several narrow-band filters are designed to interrogate different frequency bands. The basic principle can be seen in Fig.

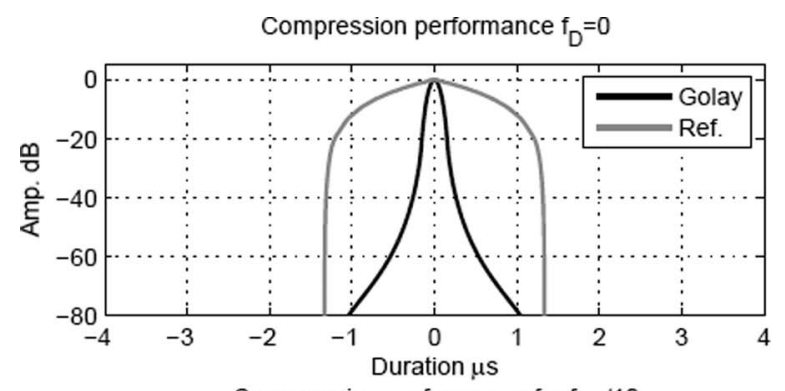

Compression performance $\mathrm{f}_{\mathrm{D}}=\mathrm{f}_{\mathrm{fpr}} / 10$

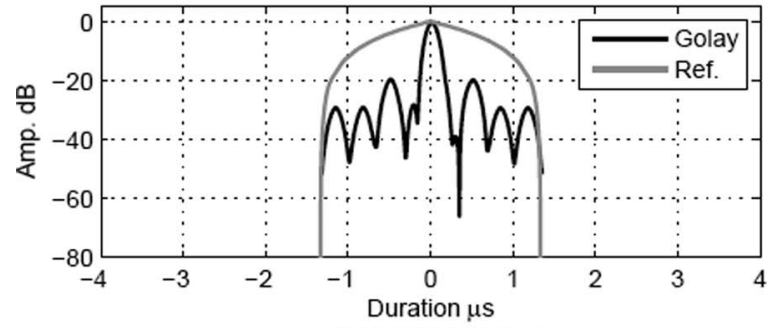

Compression performance $f_{D}=f_{\text {fpro }} / 4$

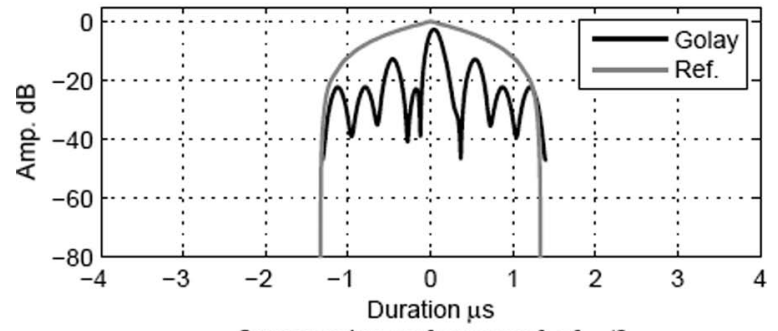

Compression performance $f_{D}=f_{\text {fprf }} / 2$

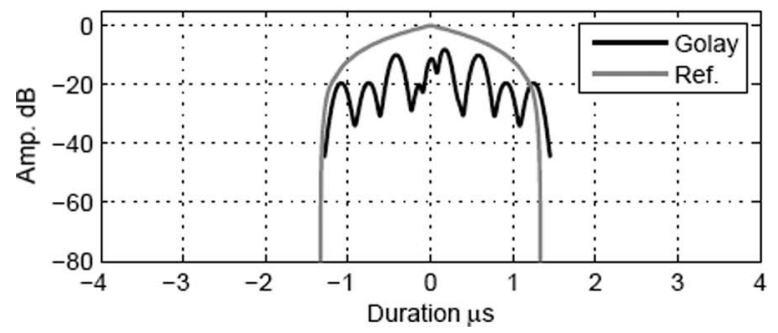

Fig. 4. The compressed code for different axial velocities. The 4 different plots describe the compressed waveform for axial velocities corresponding to $f_{D}=0$ (top plot), $f_{D}=f_{\text {prf }} / 10$ (second plot), $f_{D}=f_{\text {prf }} / 4$ (third plot), and at the aliasing limit $f_{D}=f_{\mathrm{prf}} / 2$ (bottom plot).

5 where the amplitude spectra of the filters are displayed using solid and dashed curves to facilitate visualization. Six different filters were designed as

$$
h_{f_{c}}(n)=g_{f_{c}}(n) \star g_{f_{c}}(-n),
$$

where $\star$ denotes convolution and

$$
g_{f_{c}}(n)=\sin \left(\frac{2 \pi f_{c} n}{f_{s}}\right), \quad n=0, \ldots, D
$$

with $D / f_{s}=0.89 \mu \mathrm{s}$, and the center frequencies are $f_{c}=5$, $5.5,6,6.5,7$, and $7.5 \mathrm{MHz} .{ }^{1}$ Designing the subband filters

${ }^{1}$ The reason for not using higher frequencies was that the SNR was too poor in these bands. 
can be done in many ways and is not analyzed in this paper. The important thing is that the filterbank covers the passband of the transducer so that most of the signal energy is exploited. The spectral overlap of the subband filters was chosen to use as much of the signal energy as possible. If the subband filters would have been designed to have disjoint spectral support, much of the signal energy would have been lost, which would decrease the performance of the method.

\section{B. Velocity Estimation}

The output of each filter is beamformed using dynamic receive focusing and then passed to an autocorrelation estimator. Stationary echo-canceling is performed, in-phase and quadrature channels are generated to form a complex slow-time signal by sampling the obtained data at the pulse repetition frequency. The slowly moving tissue component is removed using a mean subtraction filter with impulse response

$$
h_{s t}(k)=\delta(k)-\frac{1}{K} \sum_{u=0}^{K-1} \delta(k-u),
$$

where $K$ is the duration of the filter. The autocorrelation function in lag one was estimated for all frequency bands using $K$ slow-time samples

$$
\hat{R}_{f_{c}}(1)=\frac{1}{Q K} \sum_{q=0}^{Q-1 K-1} \sum_{k=0}^{*} r_{f_{c}}^{*}(q, k) r_{f_{c}}(q, k+1),
$$

where $r_{f_{c}}(q, k)$ is the complex slow-time signal for a given frequency band with center frequency $f_{c}$ at a depth corresponding to sample $q$ at transmission $k$. The averaging over $Q$ depths, thus, corresponds to RF-averaging and is applied to improve the quality of the estimate [19]. The axial velocity is thereafter estimated as [1]

$$
\hat{v}_{f_{c}}=-\frac{c f_{\text {prf }}}{2 \pi f_{c}} \cdot \arctan \left(\frac{\mathcal{I}\left\{\hat{R}_{f_{c}}(1)\right\}}{\mathcal{R}\left\{\hat{R}_{f_{c}}(1)\right\}}\right),
$$

where $\mathcal{I}\{(\cdot)\}$ and $\mathcal{R}\{(\cdot)\}$ are the imaginary and real parts of $(\cdot)$, respectively. The final velocity estimate is then formed by averaging over the bands (in this case at maximum 6 bands)

$$
\hat{v}=\frac{1}{N_{b}} \sum_{n_{b}=1}^{N_{b}} \hat{v}_{f_{c}\left(n_{b}\right)}
$$

where $N_{b}$ is the number of frequency bands used in the averaging and $\hat{v}_{f_{c}\left(n_{b}\right)}$ is the velocity estimate in the frequency band with center frequency $f_{c}(n b)$.

In this paper, the number of samples in the mean subtraction filter used to remove slowly moving tissue compo-

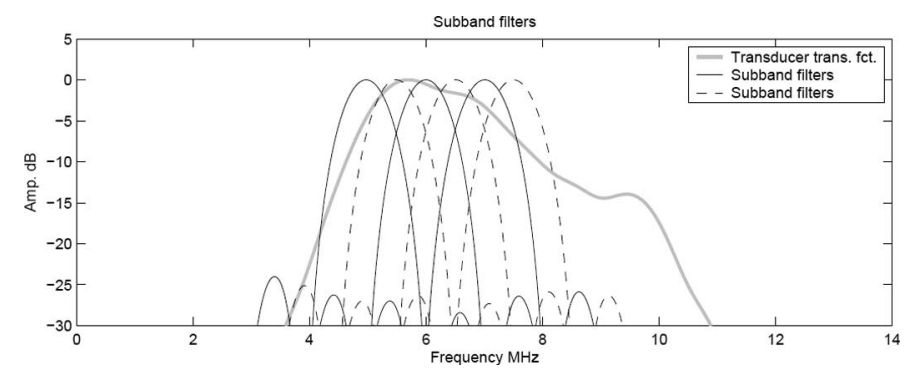

Fig. 5. Several narrow-band filters are designed to interrogate different parts of the transducer spectrum. The amplitude spectra of the filters are displayed using solid and dashed curves to facilitate visualization. The output from each filter is passed to a velocity estimator and is treated independently of the other filter outputs. This increases the number of velocity estimates per acquired data set.

nents was equal to the number of lines used to form one velocity estimate $K=32$. The number of depths used for RF-averaging was $Q=60$, corresponding to $1.11 \mathrm{~mm}$.

\section{Measurements}

\section{A. Experimental Setup}

The measurements were carried out on the experimental ultrasound scanner RASMUS [20] using a $7 \mathrm{MHz}$ linear array transducer. The sampling frequency of RASMUS is $40 \mathrm{MHz}$. The transducer had a pitch of $0.208 \mathrm{~mm}$, the kerf was $35 \mu \mathrm{m}$, and the element height was $4.5 \mathrm{~mm}$. A circulating flow rig was scanned to evaluate the performance of the different methods. The flow was laminar and had a parabolic flow-profile, and the tube radius was $6 \mathrm{~mm}$. The peak velocity in the tube was $0.09 \mathrm{~m} / \mathrm{s}$ and the Reynolds number was approximately 200. The beam-to-flow angle was $60^{\circ}$ and the center of the tube was positioned at a depth of $30 \mathrm{~mm}$ from the surface of the transducer. The transducer was submerged in the water tank when performing the measurements. A full description of the flow rig can be found in [21].

When acquiring flow data, the transducer was focused at a depth of $30 \mathrm{~mm}$ with an $\mathrm{F}$-number of 2 . The received signals were beamformed using dynamic receive focusing and a Hanning weighting over the 64 active receiving elements. The receiving aperture was centered around the data line. For the reference method and the Barker-coded experiments, the pulse-repetition-frequency $f_{\text {prf }}$ was 1890 Hz. This assured that the spectrum of the slow-time signal was not aliased. The $f_{\text {prf }}$ was $3780 \mathrm{~Hz}$ for the Golaycoded experiments to compensate for the 2 transmissions required for achieving pulse compression with this technique.

\section{B. Subband Processing}

To evaluate performance of the methods, the mean relative standard deviation (MRSTD) will be used as metric. The MRSTD (relative to the peak velocity) is defined as 

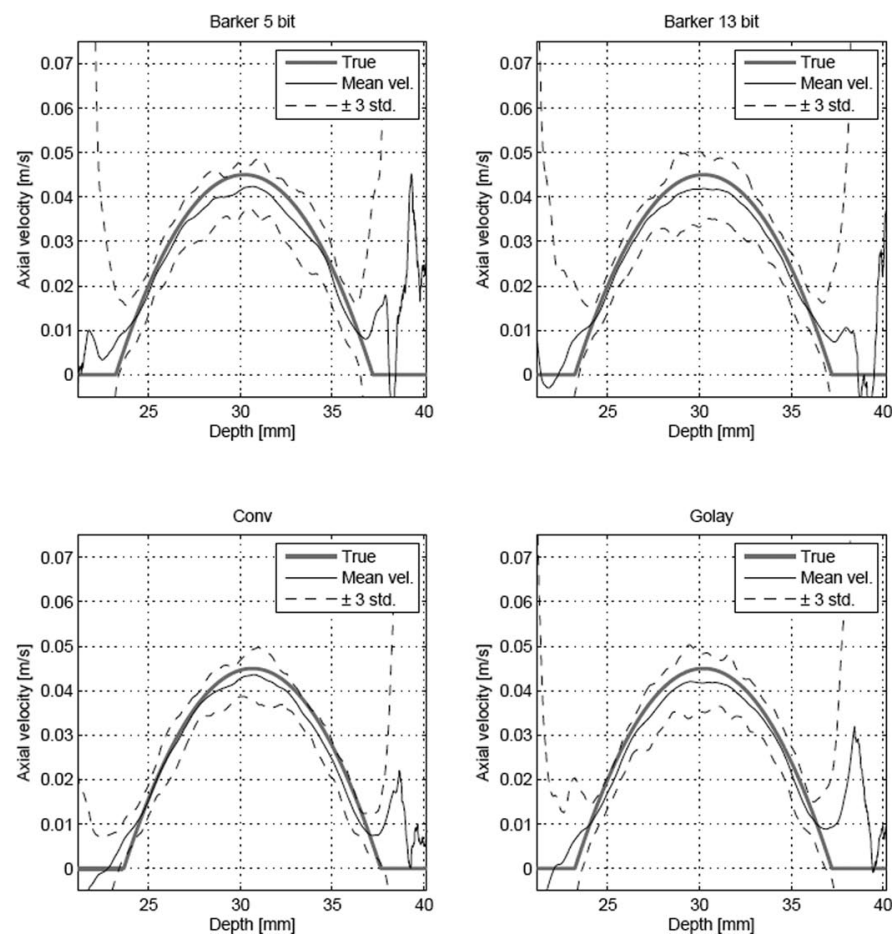

Fig. 6. Results from the flow measurement when only one band was used for the subband processing. The top left plot is the result when using the 5-bit Barker code, the top right plot is the result for the 13-bit Barker code, and the Golay-coded experiment is displayed in the bottom right plot. The reference measurement is given in the bottom left figure. The theoretical prediction is given as the thick black curve and the estimated profile is given in gray.

$$
\operatorname{MRSTD}=\frac{1}{v_{\max }}\left(\frac{1}{Z} \sum_{z=z_{1}}^{z_{2}} \sigma_{v}^{2}(z)\right)^{1 / 2} \cdot 100 \%
$$

where $\sigma_{v}^{2}(z)$ is the variance of the velocity estimate at a specific depth corresponding to sample $z, z_{1}$ and $z_{2}$ define the boundary samples for the averaging, $Z$ is the number of depths, and $v_{\max }$ is the peak velocity in the vessel. In this study, the MRTSD was evaluated over depths between 28 and $32 \mathrm{~mm}$ to avoid edge effects. The velocity was estimated for every available depth sample, so that input data for 2 consecutive depths only differed with one sample in depth. The standard deviation at depth $z$ is defined as

$$
\sigma_{v}^{2}(z)=\frac{1}{P} \sum_{p=0}^{P-1}|\hat{v}(z, p)-\bar{v}(z)|^{2}
$$

where $P$ indicates the number of velocity estimates, $\hat{v}(z, p)$ the $p$ th velocity estimate at depth $z$, and $\bar{v}(z)$ the mean velocity estimate at that depth.

The broadband data (Barker- and Golay-coded experiments) were filtered before beamforming, using the filters given in Section III-A creating 6 different sets of narrowband data suitable for velocity estimation. The narrow- band data sets were beamformed using dynamic focusing, in-phase and quadrature channels were created to generate a complex slow-time signal, and the velocity was estimated for each of the bands using the autocorrelation approach described in Section III-B. Thereafter, the velocity estimates from the different bands could be averaged to form the final estimate. First, however, only one band was used to form the velocity estimate. The result can be seen in Fig. 6, where the top left plot displays the reference method, and the other plots are the different results from the coded experiments.

Thereafter, the investigation was continued by studying the effects of averaging over more frequency bands. The velocity profiles for the experiment, when averaging over 6 frequency bands, are given in Fig. 7. When comparing Fig. 6 with Fig. 7, it can be seen that the standard deviation of the coded velocity estimates is significantly improved when averaging over several frequency bands compared with when estimating the velocity in only one frequency band. In Fig. 8, the MRSTD is shown as a function of number of frequency bands used for averaging the velocity estimates. It can be seen that the MRSTD is higher for the coded experiments when averaging over few bands compared with the reference method. However, when averaging over 6 bands, the MRSTD drops to $0.335 \%$ for the 8-bit Golay code, $0.367 \%$ for the 5-bit Barker code, and $0.310 \%$ for the 13-bit Barker code compared with the reference at $0.544 \%$. The 13-bit Barker code displays lower MRSTD as compared with the 5-bit Barker code, indicating that the better SNR provided by the longer code improves the quality of the velocity estimate.

\section{IN VIVO EXPERIMENTS}

The investigation was proceeded with in vivo measurements. The common carotid artery of a 29-year-old healthy male was scanned. The RASMUS system was used for collecting data. The same transducer as for the flow-rig experiments was used. The $f_{\text {prf }}$ was adjusted to 15 $\mathrm{kHz}$ to ensure that none of the relatively high velocities in the common carotid artery would be aliased. A color flow map (CFM) was created. This was accomplished by sliding the transmit aperture and the receiving aperture across the array while acquiring data. Each flow line was repeated 8 times before shifting the active transmit and receive aperture, and one CFM image consisted of $33 \mathrm{im}$ age lines. A CFM image could therefore be created in 17.6 ms. The number of depths used for RF-averaging was $Q=$ 60 , corresponding to $1.11 \mathrm{~mm}$. The slowly moving tissue component was removed by subtracting the mean of the 8 flow lines. A B-mode sequence was interleaved so that every ninth data transmission acquired B-mode data. The flow data were acquired using the 5-bit Barker code and a 96-sample compression filter. The B-mode data used a single cycle sinusoid and matched filtering at the receiver. All data (both flow- and B-mode) were processed using dynamic receive focusing. 

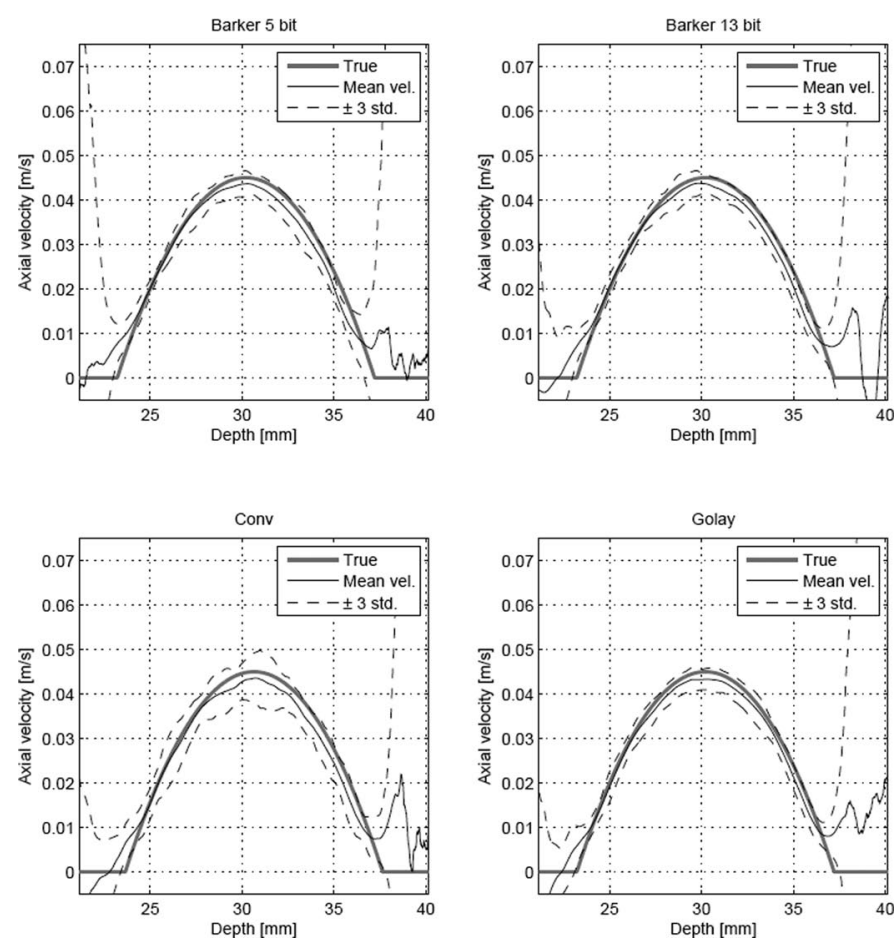

Fig. 7. Results from the flow measurement when all 6 bands are used for the subband processing. The top left plot is the result when using the 5-bit Barker code, the top right plot is the result for the 13-bit Barker code, and the Golay-coded experiment is displayed in the bottom right plot. The reference measurement is given in the bottom left figure. The theoretical prediction is given as the thick black curve and the estimated profile is given in gray.

Two of the images can be seen in Fig. 9. The image on the right shows the peak systole. All 6 frequency bands were used for the averaging. The velocities (when compensating for the angle) in the artery range from about zero to $1 \mathrm{~m} / \mathrm{s}$. In the right part of the CFM image, higher velocities can be seen as compared with the left part of the image. The image on the left is taken one frame later. Here, the flow is relatively constant across the vessel.

\section{Conclusion}

In this paper, coded excitation and subband processing has been investigated for estimating blood velocities in ultrasound. In conventional velocity estimation, a narrowband pulse is emitted, and the velocity is found using an autocorrelation algorithm. Applying a narrowband pulse has 2 advantages. First, the pulse-duration is longer, implying that the transmitted energy is increased, boosting the SNR. Second, the autocorrelation estimator yields better performance for narrowband signals.

In this paper, the full bandwidth of the transducer is interrogated by transmitting a broadband pulse. If a short excitation would be used, the SNR would drop. To compensate for this, broadband codes were used. Two coding schemes were tested, one based on complementary (Golay) codes, and one based on Barker codes. By filtering, the received signal was separated into several frequency

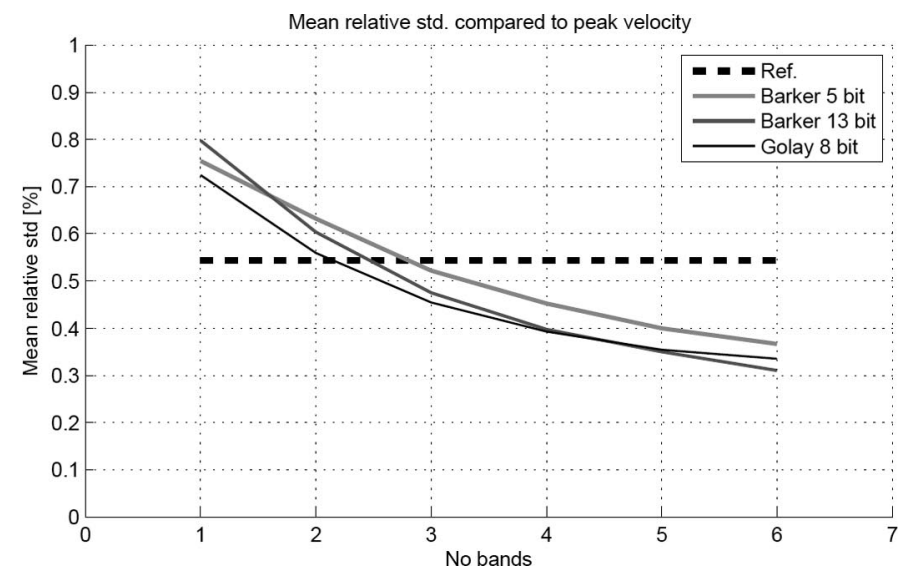

Fig. 8. The mean relative standard deviation as a function of number of frequency bands used in the velocity estimation. The reference experiment is given as the thick dashed curve, the 8-bit Golay code is indicated with dark gray, the 5-bit Barker code is shown using light gray, and the 13-bit Barker code is represented by mid-gray color.
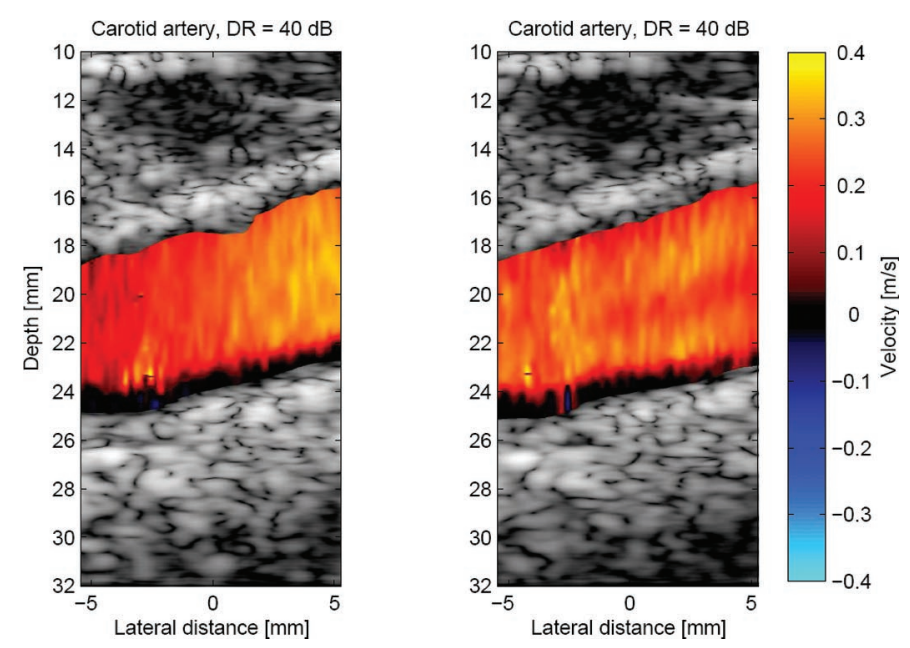

Fig. 9. The CFM image on the left is taken at the instance of the peak systole in the common carotid artery. Note the higher velocities in the right of the blood vessel. The image on the right is taken a small time instance after the peak systole. The B-mode images have a dynamic range of $40 \mathrm{~dB}$.

bands. The velocity was estimated in each of the frequency bands, then the velocity estimates were averaged to give an improved overall estimate. In the study, only bands up to $7.5 \mathrm{MHz}$ were used even though the transducer has bandwidth beyond these frequencies. This was due to the large attenuation in these bands (approximately $20 \mathrm{~dB}$ ) compared with the attenuation at the center frequency of the transducer.

The investigation was carried out on a circulating flow rig using an experimental ultrasound scanner and a commercial linear array transducer. Two 8-bit Golay codes were used to satisfy the complementary condition as given in (4). Two Barker codes were tested, with 5 and 13 bits, respectively. The Barker codes are single transmit codes and can be decoded after every emission. The Golay codes require several (in this case 2) transmissions to decode the signals. Stationarity is therefore required for the decoding 
to work without artifacts. Being sensitive to motion, one would expect that the Golay code should perform worse than the single transmit Barker code. In this study, however, no significant performance degradation was found when analyzing the quality of the velocity estimates and comparing the 2 coding schemes. This is because the flowdata lines acquired with the Golay codes are generated in the same way. Therefore, the same motion artifacts will be present in all data lines, making it possible to estimate the velocity.

The effects of coded excitation in combination with subband processing has been investigated in controlled flow-rig experiments. It was found that the MRSTD could be decreased from $0.544 \%$ to $0.310 \%$ by applying coded excitation and subband processing. It was found that the number of bands included in the velocity estimate averaging influences the MRSTD, yielding worse performance compared with the reference measurement when only one band was averaged and significant improvement when all the estimates for the different bands in the transducer passband were averaged. In the final part of the paper, in vivo experiments were carried out on a 29-year-old healthy male using the 5-bit Barker code.

\section{REFERENCES}

[1] C. Kasai, K. Namekawa, A. Koyano, and R. Omoto, "Real-time twodimensional blood flow imaging using an autocorrelation technique," IEEE Trans. Sonics Ultrason., vol. 32, no. 3, pp. 458-463, 1985.

[2] J. A. Jensen, Estimation of Blood Velocities Using Ultrasound: A Signal Processing Approach. New York: Cambridge University Press, 1996.

[3] P. R. Phillips, "Medical diagnostic ultrasound method and apparatus for improving Doppler processing," U.S. Patent 6179781, Jan. $30,2001$.

[4] J. Udesen, F. Gran, and J. A. Jensen, "A frequency splitting method for CFM imaging," in Proc. IEEE Ultrason. Symp., 2006, pp. 20192022 .

[5] R. E. Millett, "A matched-filter pulse-compression system using nonlinear FM waveform," IEEE Trans. Aerosp. Electron. Syst., vol. AES-6, no. 1, pp. 73-78, 1970

[6] M. Pollakowski and H. Ermert, "Chirp signal matching and signal power optimization in pulse-echo mode ultrasonic nondestructive testing," IEEE Trans. Ultrason. Ferroelectr. Freq. Control, vol. 41, no. 5, pp. 655-659, 1994.

[7] F. Gran and J. A. Jensen, "Designing waveforms for temporal encoding using a frequency sampling method," IEEE Trans.Ultrason. Ferroelectr. Freq. Control, vol. 54, no. 10, pp. 2070-2081, 2007.

[8] F. Gran and J. A. Jensen, "Designing non-linear frequency modulated signals for medical ultrasound imaging," in Proc. IEEE Ultrason. Symp., 2006, pp. 1714-1717.

[9] FDA information for manufacturers seeking marketing clearance of diagnostic ultrasound systems and transducers," U.S. Food and Drug Administration, Center for Devices and Radiological Health, Washington, DC, technical report, 1997.

[10] M. I. Skolnik, Introduction to Radar Systems. New York: McGrawHill, 1980

[11] J. W. Cooley and J. W. Tukey, "An algorithm for the machine calculation of complex Fourier series," Math. Comput., vol. 19, no. 90, pp. 297-301, 1965.

[12] J. W. Cooley, P. A. W. Lewis, and P. D. Welch, "Historical notes on the fast Fourier transform," IEEE Trans. Audio Electroacoust., vol. AU-15, no. 2, pp. 76-79, 1967.

[13] R. Y. Chiao and X. Hao, "Coded excitation for diagnostic ultrasound: A system developer's perspective," IEEE Trans. Ultrason. Ferroelectr. Freq. Control, vol. 52, no. 2, pp. 160-170, 2005.
[14] B. Haider, P. A. Lewin, and K. E. Thomenius, "Pulse elongation and deconvolution filtering for medical ultrasonic imaging," IEEE Trans. Ultrason. Ferroelectr. Freq. Control, vol. 45, no. 1, pp. 98-113, 1998.

[15] S. Haykin, Adaptive Filter Theory. New York: Prentice-Hall, 2002.

[16] M. O'Donnell, "Coded excitation system for improving the penetration of real-time phased-array imaging systems," IEEE Trans. Ultrason. Ferroelectr. Freq. Control, vol. 39, no. 3, pp. 341-351, 1992.

[17] H. Zhao, L. Y. L. Mo, and S. Gao, "Barker-coded ultrasound color flow imaging: Theoretical and practical design considerations," IEEE Trans. Ultrason. Ferroelectr. Freq. Control, vol. 54, no. 2, pp. 319-331, 2007.

[18] R. Y. Chiao and L. J. Thomas, "Synthetic transmit aperture using orthogonal Golay coded excitation," in Proc. IEEE Ultrason. Symp., 2000, pp. 1469-1472.

[19] T. Loupas, J. T. Powers, and R. W. Gill, "An axial velocity estimator for ultrasound blood flow imaging, based on a full evaluation of the Doppler equation by means of a two-dimensional autocorrelation approach," IEEE Trans. Ultrason. Ferroelectr. Freq. Control, vol. 42 , no. 4 , pp. $672-688,1995$.

[20] J. A. Jensen, O. Holm, L. J. Jensen, H. Bendsen, S. I. Nikolov, B. G. Tomov, P. Munk, M. Hansen, K. Salomonsen, J. Hansen, K. Gormsen, H. M. Pedersen, and K. L. Gammelmark, "Ultrasound research scanner for real-time synthetic aperture image acquisition," IEEE Trans. Ultrason. Ferroelectr. Freq. Control, vol. 52, no. 5, pp. 881-891, 2005.

[21] S. I. Nikolov and J. A. Jensen, "In-vivo synthetic aperture flow imaging in medical ultrasound," IEEE Trans. Ultrason. Ferroelectr. Freq. Control, vol. 50, no. 7, pp. 848-856, 2003.

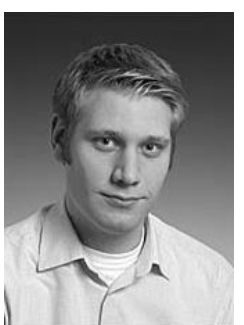

Fredrik Gran earned his M.Sc. in engineering physics from Lund University in 2002. In 2005, Dr. Gran received his Ph.D. degree from the Technical University of Denmark for work on ultrasound signal processing. From 2005 to 2008 he was employed as assistant-professor at the Technical University of Denmark. In January 2008, Dr. Gran joined GN ReSound A/S as a research scientist in hearing aid signal processing. His research interests include adaptive signal processing, adaptive beamforming, and acoustics.

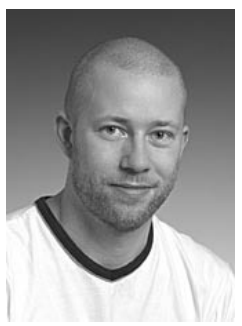

Jesper Udesen was born in 1971 and earned his M.Sc. degree in mathematics and physics from Roskilde University Center, Denmark, in 2002. He received his Ph.D. degree in 2006 from the Technical University of Denmark for work on blood vector velocity estimation. From 2006 to 2007 he was employed as a postdoctoral researcher at the University Hospital of Copenhagen. Since 2007, he has been employed at the Technical University of Denmark as an assistant professor. His research and beamforming techniques. interests include blood vector velocity estimation

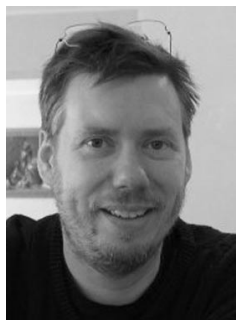

Michael Bachmann Nielsen was born in Copenhagen in 1959. He earned his M.D. degree from Copenhagen University in 1985. He served a consultancy in the Department of Radiology, Rigshospitalet (the National Danish University Hospital), in 1998. He became a full professor in 2008 and chair of the imaging research group in the Department of Radiology, Rigshospitalet. He is the editor of Ultraschall in der Medicine/European Journal of Ultrasound and chairman of the European Federation of Societies for Ultrasound in Medicine and Biology publication committee. 


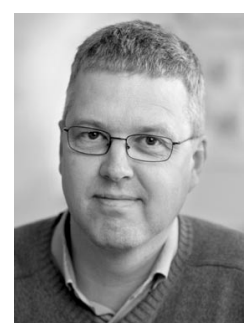

Jørgen Arendt Jensen earned his M.Sc. degree in electrical engineering in 1985 and the Ph.D. degree in 1989, both from the Technical University of Denmark. He received the Dr.Techn. degree from the university in 1996. He has published more than 160 journal and conference papers on signal processing and medical ultrasound and the book Estimation of Blood Velocities Using Ultrasound, which was published by Cambridge University Press in 1996. He is also developer of the Field II simulation program. He has been a visiting scientist at Duke University, Stanford University, and the University of Illinois at Urbana-Champaign. He is currently full professor of biomedical signal processing at the Technical University of Denmark in the Depart- ment of Electrical Engineering and head of the Center for Fast Ultrasound Imaging and the section for biomedical engineering. He is also adjunct full professor on the faculty of health sciences at the University of Copenhagen. He has given courses on blood velocity estimation at both Duke University and University of Illinois and teaches biomedical signal processing and medical imaging at the Technical University of Denmark. He has given several short courses on simulation, synthetic aperture imaging, and flow estimation at international scientific conferences. He has received several awards for his research. He is also the coorganizer of a new biomedical engineering education offered by the Technical University of Denmark and the University of Copenhagen. His research is centered around simulation of ultrasound imaging, synthetic aperture imaging, vector blood flow estimation, and construction of ultrasound research systems. 